\title{
Le Problème du comique dans La Secrétaire particulière de Jean Pliya
}

\author{
Ben Jukpor
}

Université de Brandon, Manitoba

$\mathrm{L}$

orsque Jean Pliya décrit sa pièce, La Secrétaire particulière, ${ }^{1}$ comme une comédie, il est probable qu'il conçoit le terme de comédie ${ }^{2}$ dans son acception populaire où il désigne surtout une pièce thêâtrale faite pour susciter le rire ou pour mettre en relief le caractère plaisant des personnages. Dans une comédie, les événements sont souvent présentés de manière à provoquer l'amusement ou à susciter le rire. ${ }^{3}$ Une comédie serait donc une pièce qui fait rire, c'est-à-dire une pièce plaisante, comique. ${ }^{4}$

Or le problème qui se pose en ce qui concerne La Secrétaire particulière est celui de savoir ou de déterminer dans quelle mesure cette pièce de Jean Pliya est véritablement une pièce comique, une pièce qui fait rire. Ce problème devient de plus en plus difficile à résoudre si l'on pense au fait que le rire, comme l'ont bien fait remarquer beaucoup de théoriciens du rire, et notamment Lucien Fabre, revêt un caractère d'une "extrême diversité. ${ }^{5}$ C'est en envisageant la forme peu homogène du rire que Lucien Fabre évoque kle rire de la joie, le rire du chatouillement, le rire du plaisir, le rire de l'humour, le rire du comique. ${ }^{6}$

Toutes ces formes du rire relevées par Lucien Fabre ont pourtant, à proprement parler, un trait qui se retrouve, dans une certaine mesure, d'une forme à l'autre: l'élément du plaisir ou de l'amusement. C'est pourquoi, bien que la remarque de Lucien Fabre soit pertinente, les traits distinctifs des catégories du rire évoquées par lui demeurent flous.

Beaucoup d'autres théoriciens ${ }^{7}$ distinguent deux formes particulières du rire: le rire de la joie ou du plaisir qui consiste, en général, à rire avec quelqu'un, et le rire amer, le rire de la critique - "le ris malin, le perfidum ridens, " "le comique de satire. ${ }^{9}$ Ce rire est "âpre et corrosif,,${ }^{10}$ et il consiste cette fois à rire de quelqu'un. C'est ce rire que Charles Mauron appelle "le haut comique" qui "tend au sérieux. puis au grave," et s'oppose au "bas comique" qui provoque un "rire franc."

Dans le rire de la joie, d'après les théoriciens, on rit parce qu'on est heureux. et le rire ne revêt ici aucune intention foncièrement critique. 
Dans le rire de la satire, au contraire, le rire est une arme de la critique, une manière d'attaquer celui dont le comportement est désapprobable. Pour nous, ce rire, à proprement parler, ne saura donc avoir pour but le plaisir en soi, car il naît d'une situation intenable difficilement compatible avec le plaisir. En effet, Voltaire observe que dans le fond ce "ris malin" n'est pas un vrai rire. "C'est huer plutôt que rire. ${ }^{12} \mathrm{Et}$ il faut le souligner, le rire de la satire n'a rien à voir avec l'épanouissement joyeux du sujet "riant." C'est plutôt une réaction aigre de désapprobation, une attitude critique par laquelle le sujet "riant" décrie celui qui provoque sa réaction.

En plus de ces deux formes particulières du rire - rire de la joie et rire de la satire - nous constatons une troisième forme, le rire marginal qui pourrait se prêter à la théorie bergsonienne. Pour Henri Bergson, le rire naît de la raideur, c'est-à-dire de l'"image du mécanique plaqué sur du vivant." ${ }^{13}$ Ici, celui qui fait rire le fait dans la mesure où il agit comme s'il n'avait plus aucune souplesse humaine, et comme s'il était transfiguré en chose.

Ce rire comporte-t-il une intention critique, ou est-il un rire de la joie? En fait, nous y reconnaissons dans une certaine mesure un élément du plaisir passager et une intention critique.

Le plaisir provient de la bizarrerie du comportement de l'homme-mécanique et s'explique à travers la subite prise de conscience par le rieur de sa supériorité à cet homme-mécanique, car il se trouve précisément dans une situation de bien saisir, et même d'analyser l'incongruité inhérente à l'acte de l'homme-mécanique. En même temps, il sait, ou il veut faire croire par le rire du plaisir qui nait ici de la conscience de sa supériorité, qu'il est incapable d'agir comme le fait l'hommemécanique. C'est ainsi que l'élément du plaisir que produit le rire qui nait de l'image de la raideur se double d'une intention critique. Celle-ci souligne l'infériorité de celui qui la provoque et le somme en même temps d'agir comme il faut.

Cependant, l'intention critique du rieur n'est pas aussi profonde que celle qui naît d'une situation où l'acte de l'objet du rire constituerait une menace sérieuse au bien-être ou à l'existence du rieur. En fait, l'acte de celui qui se transforme en une sorte de mécanique ne menace pas dans le fond le rieur, et il pourrait, dans certains cas, ne nuire qu'au personnage lui-même. Tel est par exemple le cas des monomanes qui sont trop obsédés par leurs propres pensées pour pouvoir donner leur attention à quelque chose d'autre. Tel est aussi le cas d'un homme qui, tellement absorbé par une pensée qui agite son esprit, ira, les yeux grands ouverts, et en plein midi, se heurter à un camion en stationnement. 
Le rire marginal qui provient de la raideur pourrait se manifester aussi lorsqu'on entreprend un acte avec une rigidité portée à l'excès. Nous pensons surtout ici à La Farce du cuvier de l'époque médiévale française où, pour punir sa femme trop exigeante, Jaquinot décide de suivre avec rigidité la liste des besognes journalières que sa femme lui a établie. Or lorsque la femme tombe dans une cuve et appelle son marl au secours, Jaquinot ne fait que consulter sa liste des besognes pour ensuite déclarer qu'il n'y est pas question de sortir sa femme d'une cuve.

Dans ces trois exemples que nous venons de donner du rire quil naitrait de la raideur - cas des monomanes, celui de l'homme qui se cogne à un camion, La Farce du cuvier - les personnages n'ont plus leur souplesse humaine, et ils se transforment en une sorte de mécanique. Cette transformation produit une situation incongrue qui, elle-même, est accueillie par un témoin désintéressé, un observateur involontaire, par un rire qui s'impose comme un acte brusque et irréfléchi. C'est un rire, ou plus précisément une réaction par laquelle on veut indiquer à celui qui provoque cette réaction de faire attention à son comportement, c'est-à-dire de reprendre sa souplesse humaine. Mais il ne s'agit pas dans ce rire, à proprement parler, d'une réaction de la joie ou du plaisir.

C'est pourquoi on pourrait effectivement dire que le rire qui nait de l'wimage du mécanique plaqué sur du vivant" est un rire marginal. C'est un rire qui se situe entre la joie franche et le déplaisir pur. Ce n'est pas particulièrement un rire de la joie. Il ne fait pas partie non plus, d'une manière profonde, du rire de la satire.

\section{$\nLeftarrow \nLeftarrow$}

Notre étude du comique dans La Secrétaire particulière s'établira, dans la mesure du possible, sur les trois formes du rire que nous venons d'évoquer: le rire de la joie, le rire de la satire, le rire marginal.

La pièce de Jean Pliya suscite-t-elle le rire de la joie, un rire où n'existe pas l'intention critique? Nous ne le pensons pas, et il nous paraît même évident que Jean Pliya conçoit sa plèce comme un moyen de critiquer, en les exposant, la corruption et l'abus de pouvoir qui règnent suprêmes en Afrique contemporaine, et qui mettent en question l'existence même du continent. Au début de sa pièce, Jean Pliya souligne ce fait et évoque la nature sérieuse du sujet abordé dans sa pièce, lorsqu'il écrit: 
Je dédie cette pièce de thêâtre à la mémoire [...] de tous les Africains victimes de leur légitime désir de travailler et de s'épanouir dans une société juste, pacifique où l'homme vive libre et ne solt pas un loup pour l'homme (p. 4).

D'une manière détournée, Jean Pliya indique par là que la société africaine dont il est question dans sa pièce n'est pas juste ou pacifique car l'homme y établit son bonheur sur le malheur d'autrui. C'est ainsi que les gens comme Chadas chez qui la corruption sociale est la seule règle de conduite considèrent toute la société comme le bien privé à exploiter à leur gré. C'est là le sujet principal de La Secrétaire particuliêre: l'exploitation de l'homme par l'homme. Un tel sujet, quelle que soit la manière dont il est présenté, n'est guère propre à susciter un rire franc, du moins chez les spectateurs africains, c'est-a-dire le peuple en général, qui, en Afrique contemporaine, paraît comme la victime éternelle de ceux qui détiennent le pouvoir.

C'est pourquoi pour nous la pièce de Jean Pliya n'a, dans le fond, rien à voir avec le rire de la joie. Pour les spectateurs africains à qui s'adresse largement la pièce, le sujet est trop sérieux pour provoquer le rire franc. ${ }^{14}$ Les spectateurs reconnaissent dans la pièce non pas un fait fictif mais une situation réelle qu'ils vivent avec le plus grand mécontentement car elle rend leur existence presque impossible.

Or, si La Secrétaire particulière ne suscite guère le rire de la joie, provoque-t-elle, d'autre part, le rire marginal, un rire qui n'est foncièrement ni celui de la joie, ni particulièrement celui de la satire?

En fait on trouve dans la pièce des traits du rire marginal mais ces traits sont très peu nombreux et ne sauront constituer un aspect important de la pièce. En plus, ils paraissent introduits dans la pièce par le dramaturge comme une manière de décharger l'atmosphère lourde d'hostilités et de malaise général dans laquelle s'inscrit la pièce.

Les traits du rire marginal proviennent ici, en particulier, de l'image de la raideur. Nous en retenons trois occasions.

La première occasion est fournie par Chadas, le personnage principal de la pièce. L'imitation rigide jusqu'à la servitude, par le personnage, du mode d'habillement européen fait de lui une sorte de mécanique dépourvu de souplesse humaine. C'est précisément le rire marginal qui se produit chez le spectateur lorsqu'il voit Chadas habillé de complet européen. Bien qu'il étouffe de chaleur il refuse d'enlever sa veste: 
Ouf J'ai chaud. Si je pouvais seulement enlever ma veste. Hélas! ce serait incorrect. Un patron doit donner le bon exemple jusqu'à l'héroïsme inclus. Certains me diront esclave des manières de Blancs. Bah! J'en ai entendu d'autres (II, 3, p.35).

En effet, pour pouvoir se décrire comme étant correctement habillé, Chadas n'enlèvera ni sa veste ni son noeud papillon malgré la chaleur qui l'accable. Le personnage finit ainsi par se révêler au spectateur comme l'kimage du mécanique plaqué sur du vivant."

La même raideur apparaît aussi chez le militaire, un ancien combattant, et donne lieu au rire marginal. Bien que l'ancien combattant ne soit plus ni dans l'armée ni véritablement soldat, il ne parvient plus à écarter les habitudes militaires ni de son habillement ni de son langage. Son "numéro matricule 27.84.75" revient sans cesse dans tout ce qu'il dit, comme une formule cabalistique complètement inutile et pleinement déplacée. Quoique maintenant civil, l'ancien combattant est régi par son ancien comportement militaire, et pour lui il est impossible d'agir autrement. C'est ainsi que le personnage apparait comme une sorte d'hommemécanique, déterminé par la raideur.

La dernière occasion du rire marginal provenant de l'image de la raideur nous est présentée par le planton. Nous pensons surtout ici à la façon dont le planton, incapable de distinguer entre les trois synonymes du mot toilette que lui fournit Chadas, ramène les trois synonymes en un seul mot. Et chaque fois qu'il parle de toilette, le planton emploie les trois synonymes en même temps. C'est ainsi que "petit coin ou cabinet ou mieux encore "vatère-clozette" " (I, 3, p. 21) de Chadas devient chez le planton "petit coin-cabinet-clozette" (Ibid.), les trois mots ne formant qu'un seul pour lui.

La raideur du personnage ne vient pas seulement de son incapacité de distinguer entre les trois mots. Elle nait aussi de la constance avec laquelle il les ramène en un seul mot, et répète sa bévue.

Cependant, l'aspect important de La Secrétaire particulière ne consiste ni en le rire marginal que provoque la raideur des personnages, ni en le rire franc qui parait presque déplacé dans la pièce. Il consiste fondamentalement en le rire de la satire qui est pour nous une critique tenace d'une situation sociale jugée inacceptable. Cette situation sociale se manifeste surtout à travers la corruption qui a lieu dans les bureaux africains, et La Secrétaire particulière est une pièce où "les moeurs 
bureaucratiques caractérisées par la corruption, l'incompétence, l'abus de pouvoir, le favoritisme, la négligence, la légèreté ${ }^{15}$ sont dépeintes avec aigreur.

L'action dans la pièce se déroule presque tout entière dans le bureau de Chadas et elle consiste à exposer les abus qui ont lieu dans ce bureau. Le bureau lui-même est conçu par le dramaturge comme représentant, en abrégé, toute l'Afrique urbanisée, de sorte que les moeurs bureaucratiques renvoient aussi aux moeurs qui ont cours en Afrique urbanisée en général. Chadas, chef de service, est conscient de ce fait, et lorsqu'il parle de rétablir l'ordre dans son bureau, il pense à instaurer la probité dans tout le pays.

En fait, le bureau de Chadas est un capharnaüm où règne "le laisser-aller à tous les niveaux," (I, 2, p. 12) comme le constate le personnage lui-même. Il ajoute qu'(il y a trop de négligence dans le pays," et compte faire de ses employés qui sont largement incompétents, "des fonctionnaires modèles" (Ibid.). L'incompétence des employés proviendrait du fait qu'étant tous protégés par des personnages importants, ils croient que leurs postes leur appartiennent de droit.

Or puisqu'ils ont leur emploi grâce à la protection des personnages importants, ils croient aussi que cette protection s'étend à leur incompétence. C'est ainsi que Jean Pliya conçoit la société africaine contemporaine, telle que la caractérisent les fonctionnaires qu'il dépeint, comme un monde qui ne va pas droit. Vu que dans la société les valeurs sociales sont renversées, le désordre remplaçant l’ordre, le dêsir exprimé par Chadas de rectifier la situation paraît déjà en lui-même déplacé et contradictoire. D'abord parce que Chadas lui-même occupe son poste grâce à la protection dont il jouit auprès d'un ministre, c'est-à-dire que son poste a été acquis et est maintenu par la corruption sociale. Il souligne ce fait avec arrogance:
[...] aujourd'hui je suis haut perché et je sais commander. Avoir des relations, être malin et débrouillard, en cela consiste l'habileté suprềme $(\mathrm{I}, 2, \mathrm{p} .16)$.

Ensuite parce que tout comme ses employés, Chadas n'est guère compétent et ne vient au bureau que pour organiser des fêtes et des pique-niques au téléphone, et surtout pour séduire ses employées et les jeunes filles qui cherchent du travail.

Or, si Jean Pliya insiste sur les paroles doucereuses de Chadas, où le personnage fait remarquer les abus qui pullulent dans le bureau, et déclare qu'il redressera la situation, c'est pour faire bien voir les contradictions entre ces paroles 
et le comportement réel du personnage. Chadas n'a nullement l'intention de mettre de l'ordre dans son bureau comme il le prétend. En fait toute la pièce est bâtie sur le dévoilement de la mauvaise foi du chef de bureau qui ne cesse de faire du zèle pour la justice en même temps qu'il perpétue l'injustice et sème la corruption. C'est précisément ces méfaits que le dramaturge censure avec acharnement chez le personnage.

En tant que chef de service, Chadas est le représentant le plus authentique des gens corrompus de la société. Mais la corruption sociale qui règne dans son bureau s'étend, à l'exception près de Virginie, à tous les employés, même au planton. Pour mieux faire remarquer cette corruption, le dramaturge présente un paysan qui est comme affolé et terrorisé par l'irresponsabilité et par la dépravation des employés, ainsi que par l'attitude méprisante à l'égard d'autrui qui caractérise les citadins africains. ${ }^{16}$ Désenchanté de ce mode de vie urbain, le paysan l'oppose à la bonne foi et à la sollicitude pour autrui qui dominent dans son village.

C'est ainsi qu'après avoir passé deux jours d'attente inutile devant le bureau de Chadas, et après avoir deux fois payé les frais de voyage, de son village à la ville, où il doit obtenir l'acte de naissance qui lui permettra d'envoyer son enfant à l'école, le paysan constate, la mort dans lâme, que dans cette société de l'Afrique contemporaine rien ne marche plus comme il faut. Puisqu'il n'est qu'un simple paysan, c'est-à-dire qu'il n'est rien du tout, selon le raisonnement des fonctionnaires, on ne le laissera voir le chef de bureau qu'après tout le monde, même s'il est le premier à se présenter devant le bureau de Chadas. En plus, il lui faudra acheter ce service par un pourboire qu'il n'est nullement en mesure de donner à qui que ce soit. Autrement dit, il ne parviendra jamais à obtenir l'acte de naissnace qu'il vient chercher en ville.

Son affolement va grandissant à travers les diverses altercations qu'il a avec la grande dame de ville, la jeune fille, le militaire, et finalement le planton. Ces altercations se soldent par le désespoir et par la déception du paysan à l'égard de la société africaine post-coloniale. A la suite de sa déception, le paysan finit par comprendre que la société post-coloniale de l'Afrique urbanisée est un monde à l'envers. ${ }^{17}$

Etant donné que l'univers urbain est un monde qui ne va pas droit, il n'est pas étonnant que le paysan fasse remarquer son admiration pour les Français au militaire qui se vante d'avoir lutté pour ceux-ci: 
J'aime beaucoup les Français. Je me plaignais déjà de leur temps. Mais ce que je vois aujourd'hui est trop dur pour moi (II, 4, p. 43-44).

Il va jusqu'à souhaiter leur retour:

[...] Toi [c'est-à-dire le militaire] qui connais les secrets des Blancs, quand reviendront-ils et quand finira l'indépendance? (Ibid., p. 4 4).

Et lorsque le planton lui dit qu'il ne le laissera pas passer à moins qu'il ne lui donne un pourboire - "Si tu tiens à voir le patron, ne viens jamais ici les mains vides [...]. ${ }^{18}$ Pas de cadeau, pas de planton" (Ibid., p. 45) - le paysan, déjà ébahi, s'en va définitivement, bien convaincu cette fois que sa déception est sans remède:

Ah! qui me disait qu'avec le départ des Blancs le temps de la justice est venu? (S'en allant) Tant pis pour moi. Tant pis pour le monde (Ibid., p. 46).

Ainsi le paysan est progressivement amené jusqu'à l'aliénation du moi à travers la dégradation presque divinisée des moeurs qu'il constate chez les citadins. Ce refus du moi n'est pas le même que celui d'un Thôgô-gnini ${ }^{19}$ ou d'un Chadas. Si ceux-ci, par orgueil et par le désir d'être considérés comme des personnages importants, rejettent les valeurs africaines au profit des moeurs occidentales, perpétuant ainsi la mainmise de l'Europe sur l'Afrique, le paysan, quant à lui, souhaite le retour des Blancs en désespoir de cause. Il conçoit ce retour comme un moyen d'assouvir sa soif de justice qui a été compromise par la corruption et l'irresponsabilité de certains Africains qui étouffent tout le pays.

Dans ce monde corrompu, le planton ne fera pas son travail s'il ne reçoit pas de cadeau. Chadas met ses employés presque à rançon. Pour garder leurs places au bureau, Jacques lui donne "deux dindons et des bouteilles de boisson" (II, 1, p. 30). Virginie lui donne, malgré elle, une bouteille de whisky. Nathalie, sa secrétaire particulière, se donne régulièrement à lui et n'est plus pour lui qu'un objet de plaisir sexuel. En outre, Chadas n'hésitera pas à créer pour une autre jeune fille une nouvelle place de dactylo dans son service, pourvu qu'elle se livre à lui. Il observe 
méchamment qu'il a déjà trois dactylos où une seule suffit. Il intimide Avocè Holonon, le chômeur que sa voiture renverse et blesse gravement et lui donne 10.000 francs pour l'empêcher de dire la vérité sur les circonstances de l'accident. Denise, l'avocate d'Avocè est finalement bafouée par un Chadas tout plein de lui-même.

Eu égard à cette corruption qui se déroule dans une large mesure au bureau de Chadas, il est évident que le bureau, tel que le conçoit le dramaturge, n'est point un lieu où l'on s'occupe d'intérêts du pays mais un endroit où l'on assure la destruction de celui-ci. La perversion des moeurs telle qu'elle se manifeste dans le bureau de Chadas n'est pas un cas isolé. Elle renvoie à la situation dégradante dans laquelle se trouvent les services publics dans certains Etats de l'Afrique contemporaine. Comme l'indiquent les éditeurs CLE,

La Secrétaire particulière [...] s'enracine très profondément dans la vie actuelle [...] Elle est surtout le cri de tous les Africains victimes de leur désir de travailler et de s'épanouir dans une société juste. $^{20}$

Ce cri des Africains victimes de la perversion des employés qui paralyse la société se fait entendre surtout chez le paysan où il paraît dans toute sa vigueur comme un véritable cri de détresse. La détresse du paysan est en fait celle du peuple en général, qui, face à cette Afrique que les employés étranglent, n'hésite plus à implorer le secours des Blancs.

Ainsi si le paysan souhaite le retour des Blancs, c'est qu'il ne voit pas d'autre moyen de détruire l'avilissement où sombre le pays. D'où il suit que le refus même du moi chez le paysan, c'est-à-dire sa révolte désespérée contre l'abus perpétré par ses compatriotes, est avant tout dicté par le souci pour le salut du pays. Il s'agit pour lui de remplacer le moi libre mais malade et mourant par le moi enchainé mais "sain," dans la mesure où l'Afrique dominée par l'Europe est aussi l'Afrique qui porte toujours les chaînes de l'esclavage.

Mais le paysan se trompe en croyant qu'il suffit de rappeler les Blancs, c'est-à-dire d'asservir de nouveau l'Afrique pour assainir la société. Celui qui est enchaîné n'est nullement sain car il est mis dans l'état de souffrance double: souffrance morale, puisqu'il est dépersonnalisé; souffrance physique, puisqu'il est réduit en esclavage. Des deux côtés - celui d'homme libre et celui d'homme 
enchaîné, - 11 y a toujours le malheur social que le paysan, plus précisément le peuple, est obligé de souffrir.

Ce que le dramaturge expose par là c'est la situation de ruine sociale dans laquelle se trouvent plusieurs Etats de l'Afrique contemporaine, et plus précisément le Bénin, où se situe l'action de la pièce. Dans ce pays, il ne parait plus possible de marcher en avant, c'est-à-dire d'instaurer une forme de progrès social digne de ce nom, à cause de la corruption qui détruit le pays. D'autre part, revenir en arrière, c'est-à-dire rappeler les oppresseurs blancs, équivaudra à replonger le pays dans l'ancien gouffre. Ainsi tout le pays est comme suspendu entre Charybde et Scylla où tout mouvement paraît interdit car il comporte une forme de désastre.

Cette stagnation du pays est bien mise en évidence par la situation dans laquelle se trouve la jeune fille qui est prête à tout donner, même elle-même, afin de pouvoir nourrir ses quatre petits frères et une mère malade, abandonnée par le mari; et encore par le malheur du militaire chômeur. Le militaire et sa famille sont réduits à n'être que le symbole éloquent de la misère. Le paysan qui ne parvient pas à obtenir lacte de naissance de son enfant, la jeune fille "prête à tout," le militaire, sont tous victimes de la société qui est tombée dans le marasme. Grâce à eux, le dramaturge souligne la misère dans laquelle est plongé tout le pays en décriant d'une manière amère les auteurs du malheur: les Chadas.

Ce malheur se prolonge et se cristallise, symboliquement, dans la grossesse inacceptable de la secrétaire particulière qui grandit, arrogante, de la même manière que pousse, insolente, un bouton démesuré et défigurant sur le front d'une femme d'une laideur déjà agressive. Tout comme cette femme ne saura considérer sa situation amusante, les Africains ne sauront trouver plaisante la situation dans laquelle est l'Afrique, telle que la présente Jean Pliya, à travers la dépravation des moeurs qui caractérise les services publics. La situation qu'expose La Secrétaire particulière, qui est précisément celle de la dégénérescence dans laquelle est mise l'Afrique indépendante ne suscite ni le rire de la joie ni le rire marginal.

La Secrétaire particulière n'est pas, à proprement parler, une pièce comique. L'étiquette de comédie que Jean Pliya colle à sa pièce est un pavois dont il se sert pour se protéger contre ceux qu'il critique, et qui, dans la vie réelle sur laquelle est calquée la pièce, détiennent en général le pouvoir. La pièce de Jean Pliya est une critique tranchante, presque à la Juvénal, des abus qui ont cours en Afrique indépendante. C'est un cri d'alarme, un cri de détresse, voire un cri d'indignation déchirant devant ce scandale que constitue pour l'esprit la déchéance morale solidement enracinée dans la société post-coloniale de l'Afrique urbanisée. 


\section{Notes}

${ }^{1}$ Jean Pliya, La Secrétaire particulière (Yaoundé: CLE, 1975).

${ }^{2}$ Robert Champigny, Le Genre dramatique (Monte-Carlo: Editions Regain, 1965), p. 147, signale l'imprécision et la confusion que recouvrent les termes comédie, tragédie et drame tels que les emploie la critique contemporaine, et observe que la division des pièces de théâtre en ces genres n'est guère pertinente. Quant à nous, l'examen générique du terme de comédie dépasse le but de notre étude présente, et nous insistons ici sur l'acception populaire du terme.

${ }^{3}$ M. Abrams, A Glossary of Literary Terms (New York: Holt, Rinehart and Winston, Inc., 1971), p. 25.

${ }^{4}$ Pierre Larousse, Nouveau Larousse universel (Paris: Librairie Larousse, 1969).

${ }^{5}$ Lucien Fabre, Le Rire et les rieurs (Paris: Gallimard, 1929), p. 36.

${ }^{6}$ Lucien Fabre, loc. cit.

${ }^{7}$ Voir notamment:

Voltaire, "Rire," Oeuwres complètes: Dictionnaire philosophique, tome VII (Paris: Lequien, Libraire, 1821), p.141.

Charles Mauron, Psychocritique du genre comique (Paris: Librairie José Corti, 1964), p.9.

René Jasinskd, Molière et le misanthrope (Paris: Nizet, 1963), p. 293.

Grand dictionnaire universel du XIXème siècle (Paris: Administration du Grand dictionnaire universel, 1875).

${ }^{8}$ Voltaire, op. cit., p. 141.

${ }^{9}$ René Jasinski, loc. cit., p. 293.

${ }^{10}$ René Jasinski, ibid.

${ }^{11}$ Charles Mauron, op. cit., p. 9.

${ }^{12}$ Voltaire, op. cit., p. 141.

${ }^{13}$ Henri Bergson, Essai sur la signification du comique (Paris: Librairie Félix Alcan, 1928), p. 58.

${ }^{14}$ Voir Christian Valbert, "Moyens de diffusion et impact sur le public: Enquête sociologique," Le Théâtre négro-africain: Actes du colloque d'Abidjan, 1970 (Paris: Présence Africaine, 1971).

${ }^{15}$ Mukala Kadima-Nzuji, "La Secrétaire particulière," Notre Librairie $\mathrm{N}^{\circ} 41$, (avriljuin 1978), p. 62. 
${ }^{16}$ Voir: Sembène Ousmane, Le Mandat, précédé de Véhi Ciosane (Paris: Présence Africaine, 1966).

Mongo Beti, Perpétue .... (Paris: Buchet/Chastel, 1974).

${ }^{17}$ Dans Papassidi, maître-escroc, les villageois réagissent de la même manière devant les moeurs bureaucratiques et décrivent la ville comme un monde déréglé. Voir: Bernard Dadié, Papassidi, maître-escroc (Dakar-Abidjan: Les Nouvelles Editions Africaines, 1975), V, p. 57.

${ }^{18}$ C'est presque à la lettre l'idée que le planton exprime dans Papassidi ... op. cit., II, p. 18, lorsqu'il oblige Papassidi à lui donner le pourboire afin de voir le commandant. Voir également: François Agouvinon, Qui a tué Sabine? (Paris: La Maison du Manuscrit, 1977), Tab. I, I, p. 7. Ici, un fonctionnaire, Gérard, après avoir passé la plupart du temps au téléphone avec des amis, déclare dans des termes clairs, à l'homme qui attend ses services dans la queue, depuis longtemps, qu'il ne saura lui rendre ces services sans pourboire.

${ }^{19}$ Voir: Bernard Dadiê, Monsieur Thôgô-gnini (Paris: Prêsence Africaine, 1970).

${ }^{20}$ Les éditeurs, La Secrétaire particulière, op. cit., p. 98. 